Published in final edited form as:

Angew Chem Int Ed Engl. 2014 May 5; 53(19): 4796-4801. doi:10.1002/anie.201309334.

\title{
Particle Display: A Quantitative Screening Method for Generating High-Affinity Aptamers**
}

\author{
Jinpeng Wang ${ }^{+}$, \\ Department of Mechanical Engineering, Materials, and Institute for Collaborative Biotechnologies, \\ University of California, Santa Barbara, CA, 93106, University of California, Santa Barbara, Santa
} Barbara, CA 93106 (USA), Fax: (+1) 805-893-8651

\section{Qiang Gong ${ }^{+}$,}

Department of Mechanical Engineering, Materials, and Institute for Collaborative Biotechnologies, University of California, Santa Barbara, CA, 93106, University of California, Santa Barbara, Santa Barbara, CA 93106 (USA), Fax: (+1) 805-893-8651

\section{Nupur Maheshwari,}

Department of Mechanical Engineering, Materials, and Institute for Collaborative Biotechnologies, University of California, Santa Barbara, CA, 93106, University of California, Santa Barbara, Santa Barbara, CA 93106 (USA), Fax: (+1) 805-893-8651

\section{Michael Eisenstein,}

Department of Mechanical Engineering, Materials, and Institute for Collaborative Biotechnologies, University of California, Santa Barbara, CA, 93106, University of California, Santa Barbara, Santa Barbara, CA 93106 (USA), Fax: (+1) 805-893-8651

\section{Mary Luz Arcila,}

Molecular, Cellular, and Developmental Bioloy, University of California, Santa Barbara, CA, 93106

Kenneth S. Kosik, and

Molecular, Cellular, and Developmental Bioloy, University of California, Santa Barbara, CA, 93106

\section{Prof. H. Tom Soh}

Department of Mechanical Engineering, Materials, and Institute for Collaborative Biotechnologies, University of California, Santa Barbara, CA, 93106, University of California, Santa Barbara, Santa Barbara, CA 93106 (USA), Fax: (+1) 805-893-8651

H. Tom Soh: tsoh@engineering.ucsb.edu

\footnotetext{
** We are grateful for the financial support of the ARO Institute for Collaborative Biotechnologies (W911F-09-D-0001), National Institutes of Health (U54 DK093467, U01HL099773-01, R01A1076899), and Department of Defense (W81XWH-09-0698). We thank Dr. Andrew T. Csordas for his technical assistance. We also would like to thank Dr. Erkki Ruolahti, Dr. Tambet Teesalu and Dr. Kazuki N. Sugahara for helpful discussions.

(c) 2013 Wiley-VCH Verlag GmbH \& Co. KGaA, Weinheim

Correspondence to: H. Tom Soh, tsoh@engineering . ucsb. edu.

+These authors contributed equally to this work.

Supporting information for this article is available on the WWW under http://dx.doi.org/10.1002/anie.201xxxxxx.
} 


\section{Abstract}

We report an aptamer discovery technology that reproducibly yields higher affinity aptamers in fewer rounds compared to conventional selection. Our method (termed particle display) transforms libraries of solution-phase aptamers into "aptamer particles", each displaying many copies of a single sequence on its surface. We then use fluorescence-activated cell sorting (FACS) to individually measure the relative affinities of $>10^{8}$ aptamer particles and sort them in a highthroughput manner. Through mathematical analysis, we identified experimental parameters that enable optimal screening, and demonstrate enrichment performance that exceeds the theoretical maximum achievable with conventional selection by many orders of magnitude. We used particle display to obtain high-affinity DNA aptamers for four different protein targets in three rounds, including proteins for which previous DNA aptamer selection efforts have been unsuccessful. We believe particle display offers an extraordinarily efficient mechanism for generating high quality aptamers in a rapid and economic manner, towards accelerated exploration of the human proteome.

\section{Keywords}

Aptamers; high-throughput screening; directed evolution; FACS; SELEX

Complete functional exploration of the proteome will require access to comprehensive sets of well-characterized affinity reagents that specifically bind to their respective target proteins with high affinities ${ }^{[1,2]}$. Accordingly, research communities in the U.S., Europe and Asia have recently mounted international efforts to generate high-quality reagents for targeting the human proteome ${ }^{[3]}$. The technical challenges are considerable; the proteome consists of multiple closely related protein variants arising from alternative splicing and post-translational modifications (PTMs), yielding complexity orders of magnitude greater than that of the genome ${ }^{[1,2]}$. Unfortunately, existing antibodies only target a small fraction of the proteome, and there is an urgent need for novel technologies that can efficiently generate reagents for the large number of unaddressed protein targets ${ }^{[2,4]}$.

Nucleic acid aptamers have garnered significant interest over the past two decades as a promising alternative to antibodies, as they are chemically synthesized and their discovery is performed completely in vitro rather than relying on in vivo biological processes, making them potentially well suited for high-throughput discovery ${ }^{[1,5,6]}$. Aptamers are also thermostable, fold reversibly and can be distributed as sequence information rather than as a physical entity, greatly accelerating reagent dissemination throughout the research community ${ }^{[1,5,6]}$. Finally, aptamers are cost-effective, and can be readily produced using standard laboratory techniques such as $\mathrm{PCR}^{[1,5,6]}$.

Despite these useful features, the number of published aptamers with sufficient affinity and specificity for proteomic analysis is extremely limited in comparison to antibodies ${ }^{[5,7]}$. Two reasons have been previously proposed to explain this shortage of high-quality aptamers. First and foremost, there is anecdotal evidence that natural nucleic acids may not possess the full spectrum of chemical functional groups and conformational space needed to yield highquality aptamers for many proteomic targets. In fact, recent reports have suggested that it 
may only be possible to generate natural DNA aptamers for less than $30 \%$ of the human proteome ${ }^{[8]}$. This problem has been addressed to some extent by adding chemical diversity via the introduction of modified nucleotides, and several efforts have already yielded aptamers with improved specificity and affinity ${ }^{[8,9]}$. The second explanation relates to inefficiencies in the aptamer discovery process ${ }^{[10]}$. Conventional aptamer discovery via SELEX requires multiple rounds of affinity-based enrichment followed by PCR-based amplification ${ }^{[10]}$. However, the efficiency of SELEX-style methods is constrained by the limited enrichment that can be achieved in a single round. Irvine and others have shown that the theoretical maximum enrichment that can be achieved for a given aptamer relative to another lower-affinity aptamer in a single round is equal to the ratio of their equilibrium dissociation constants $\left(K_{d}\right)^{[11,12]}$. For example, a high affinity aptamer with a $K_{d}$ of $1 \mathrm{nM}$ can only be enriched 100 -fold relative to another aptamer with a $K_{d}$ of $100 \mathrm{nM}$ in a single round of selection. Given that SELEX typically begins with a large, diverse library (typically in excess of $10^{12}$ molecules), this necessitates many rounds of selection (typically $8-15$ rounds), which in turn introduce undesired biases including loss of rare sequences ${ }^{[13]}$, PCR bias ${ }^{[14]}$ and parasitic amplification of low-affinity or non-specific sequences ${ }^{[11,12]}$. Accordingly, many SELEX experiments ultimately fail or yield low-quality aptamers ${ }^{[8,12]}$, and there is an urgent need for alternative aptamer discovery technologies that can overcome the fundamental limitations inherent to affinity-based selection ${ }^{[2]}$.

To this end, we describe a screening-based method for aptamer discovery (termed particle display), in which we quantitatively measure the affinity of every aptamer candidate sequence in a library and individually sort them in a high-throughput manner. Drawing inspiration from yeast ${ }^{[15]}$ and bacterial ${ }^{[16]}$ display techniques used in protein engineering, our particle display method transforms individual aptamers into aptamer particles (APs), wherein each particle presents many copies of a single nucleic acid sequence on its surface. We then individually measure the relative affinities of each of these APs via fluorescenceactivated cell sorting (FACS) and isolate only those with the highest affinities. Although FACS has been previously used for aptamer discovery ${ }^{[17]}$, our approach is distinct those in the literature, because it enables sorting of individual aptamers after measuring their affinity. In this way, particle display achieves enrichment performance that far exceeds the theoretical maximum achievable with any selection method by many orders of magnitude.

To experimentally demonstrate the effectiveness of our method, we generated high-affinity natural DNA aptamers for four different proteins within three rounds of screening, including two proteins for which previous DNA aptamer selection attempts have been unsuccessful without resorting to the use of chemically modified nucleotides ${ }^{[8]}$. These results indicate that particle display offers an effective means for generating superior aptamers, and suggest that a broader swath of the proteome may be accessible to DNA aptamers than previously envisaged.

Particle display enables us to measure the binding affinities of more than 100 million aptamers by converting a library of solution-phase aptamers into APs, and individually sorting them in a high-throughput fashion using FACS (Sch. 1). We synthesize these APs using emulsion polymerase chain reaction (ePCR) ${ }^{[18]}$ (for a detailed description, see Methods). Briefly, we prepare water-in-oil emulsions with PCR reagents, such that each 
droplet contains (in most cases) one DNA template and one magnetic bead coated with forward primer (FP) (Sch. 1, step 1). We then perform PCR amplification within the droplet, yielding particles that display $\sim 2.4 \times 10^{5}$ copies of the aptamer on their surface (Sch. 1, step 2). After breaking the emulsion and removing unreacted PCR reagents, we denature and release the reverse strands with $\mathrm{NaOH}$ and collect the APs through magnetic separation (Sch. 1, step 3). We then incubate these APs with fluorescently labelled target protein (Sch. 1, step 4). It is important to note that the fluorescence intensity from proteins captured by the APs is proportional to the binding affinity of the aptamer (see theoretical analysis below). This direct correlation between fluorescence and aptamer affinity enables us to quantitatively identify and sort the aptamers with highest affinities via FACS (Sch. 1, step 5). After FACS, we perform PCR directly from the sorted APs (Sch. 1, step 6) to generate an enriched pool of aptamers for the next round of screening or for sequencing (Sch. 1, step 7).

Particle display offers many important advantages over conventional selection. First and foremost, because each aptamer is individually measured and sorted based its binding affinity to the target protein, enrichment performance is not subject to the same theoretical upper bound that limits conventional selection. This makes it possible to achieve finer discrimination between aptamers with similar affinities, enabling isolation of the highestaffinity aptamers in far fewer rounds (see analysis below). Second, particle display can virtually eliminate unwanted enrichment of non-specific aptamers as a result of stochastic binding events. In conventional selection, a substantial percentage of low-quality aptamers is inevitably enriched due to random, non-specific binding ${ }^{[12]}$. With an AP that displays $10^{5}$ copies of the same low-affinity aptamer, however, such random binding events will yield low overall fluorescence and these APs will therefore be eliminated during FACS. Finally, FACS enables users to visualize the screening process, and thus makes it possible to finely control the stringency of the screen and thereby designate the desired aptamer affinity threshold in a quantitative and reproducible manner.

There are two experimentally adjustable parameters that govern the stringency of particle display: the concentration of the target protein and the placement of the sort gate during FACS. We used mathematical modelling to explore how these two parameters affect the screening process, and to identify the optimal values that yield the highest affinity aptamers. Since the fluorescence intensity of an AP is directly proportional to the aptamer affinity (see Eq. S1), we first sought to identify the target concentration that maximizes the difference in fluorescence signal between APs displaying aptamers with differing affinities. To do so, we calculated the ratio of the mean AP fluorescence for the highest-affinity aptamer relative to other, lower-affinity aptamers in the pool (Eq. S2, see Supplementary Information for derivation). We focused our analysis on aptamers with $K_{d}$ s that are between 2- and 10-fold higher (i.e. lower affinity) than the highest-affinity aptamer, because successful isolation of these aptamers automatically ensures the exclusion of aptamers with poorer affinity.

The resulting analysis revealed an optimal target concentration that yields the maximum difference in fluorescence between the AP with the highest affinity and the rest of the population (denoted by * in Fig. 1a). This optimal target concentration can be closely approximated as one-half of the $K_{d}$ of the highest-affinity aptamer (Eq. S3, see Supplementary Information for derivation). By substituting this target concentration into Eq. 
S1, we found that APs displaying the highest-affinity aptamers exhibit a mean fluorescence of $\sim F_{\max } / 3$ (Fig. S1a), where $F_{\max }$ is the maximum fluorescence of an AP saturated with labeled target. Taken together, these findings offer useful experimental guidelines for optimizing FACS screening; the highest-affinity aptamers in a given pool can be effectively isolated by setting the FACS sort gate at $F_{\max } / 3$ and adjusting the target concentration such that only the particles with the strongest fluorescence signal (e.g., $\leq 0.1 \%$ of the population) are collected.

These screening conditions deliver enrichment performance that far exceeds the theoretical maximum that can be achieved with affinity-based selection by many orders of magnitude, and we performed a mathematical analysis to calculate the enrichment that can be achieved with particle display. We assumed a Gaussian distribution of fluorescence intensities (in logarithmic scale) for individual AP populations in our analysis ${ }^{[19]}$. This is because any given pool of APs is likely to contain various populations of APs that display the same aptamer sequence, and the APs within these populations can exhibit a range of fluorescence intensities due to experimental variability (e.g., differences in aptamer copy number). The resulting distributions (See Fig. S1b) allowed us to determine the overlap in fluorescence among populations of APs that each display different aptamer sequences. From this result, we estimated the probability of recovering an AP displaying a given aptamer sequence based on its affinity, and used this estimate to calculate the enrichment of the highest-affinity aptamers in a round of FACS (see Supplementary Information for derivation). As discussed above, in affinity-based selection (i.e., SELEX), the theoretical maximum enrichment that can be achieved for a given aptamer relative to another, lower-affinity aptamer in a single round is equal to the ratio of their equilibrium dissociation constants; thus, aptamers with a $K_{d}$ of $100 \mathrm{pM}$ can at most be enriched 10-fold relative to a population of aptamers with $K_{d}$ of $1 \mathrm{nM}$ after a single round. By comparison, we determined that particle display can achieve $1.7 \times 10^{9}$-fold enrichment of the same aptamer population in a single round, enabling dramatically more efficient purification of high-affinity aptamers (Fig 1b).

We experimentally validated the advantages of particle display by using our method to obtain high-affinity aptamers for four different proteins - thrombin, ApoE, PAI-1 and 4-1BB - in only three rounds. We chose thrombin and ApoE in order to compare the affinity of our aptamers to previously published aptamers for these targets ${ }^{[20-22]}$. PAI-1 and 4-1BB were chosen because, although RNA aptamer have been reported ${ }^{[23,24]}$ revious attempts at generating natural DNA aptamers for these proteins via SELEX were unsuccessful and ultimately required the use of chemically modified bases ${ }^{[8,23,24]}$, suggesting that natural DNA may lack the chemical and/or structural diversity to yield useful aptamers for these two proteins.

Although one can synthesize APs from a naïve random library, due to the practical throughput limitations of our FACS instrument ( $4 \times 10^{7}$ particles per hour) we first performed one round of conventional selection using magnetic beads starting with $10^{14}$ copies of random DNA library (Round 0), and used this enriched pool to synthesize an initial AP library consisting of $\sim 10^{8}$ particles (See Methods). Prior to the screen, we used fluorescently-labeled target to measure non-specific binding to FP-coated particles. $\sim 80 \%$ of the APs after ePCR are predicted to display only the FP on their surface based on Poisson 
statistics, and thus should not exhibit significant binding to the protein targets. Thus, FPdisplaying particles serve as an excellent negative control to establish the "reference gate" for calibrating non-specific binding in the particle display screen (Fig. 2, red). In contrast, particles with higher fluorescence intensity within the sort gate (Fig. 2, green) indicate higher affinity aptamers. We performed three rounds (R1-3) of particle display screening for each of our four target proteins, and the resulting FACS plots are shown in Figure 2a. In the first round, we exerted low screening stringency to prevent potential loss of high-affinity APs. This was achieved by setting the sort gate close to the reference gate, or with some overlap, such that at least $0.1 \%$ of APs were collected (Fig. 2a, row 1). Aptamers isolated in R1 for the four target proteins were PCR amplified to synthesize the APs for R2. Since a greater number of APs displaying each aptamer sequence were present in R2 and R3, we used the optimal screening conditions described above. Specifically, we set the sort gate at $F_{\max } / 3$ and decreased the target concentration such that we collected $\sim 0.1-0.2 \%$ of the APs (Fig. 2a, rows 2 and 3). Experimental conditions for all protein targets and screening rounds are summarized in Table $\mathrm{S} 1$.

After three rounds of screening, the average affinity of the aptamer pool dramatically increased for all four target proteins. This can be readily observed from the fact that relative to the starting library, a far greater percentage of the AP population resides outside of the reference gate in R3, at a considerably lower target concentration. For example, the fraction of sorted APs for thrombin jumped from $0.1 \%$ for the initial pool (Fig. 2a) to $14.6 \%$ for the R3 pool at a 50-fold lower thrombin concentration (Fig. 2b). Given this clear increase in binding affinity, we cloned the R3 pools into E. coli (see Methods) and picked 20 aptamer clones from each pool (Table S2). We then synthesized APs displaying each sequence (Fig. S2) and measured their relative fluorescence at a fixed target concentration (see Methods) (Fig. S3). From these data, we chose the aptamers with the highest relative fluorescence for each target (Table S3), and subsequently obtained their $K_{d}$ values using a standard fluorescence-based assay ${ }^{[22]}$. We present the binding isotherms in Figure 3 and secondary structures as modelled by mfold ${ }^{[25]}$ in Figure S6.

Particle display consistently generated aptamers with exceptional affinities for all four target proteins. For thrombin and ApoE, the aptamers discovered with particle display exhibited considerably higher affinities than previously reported aptamers for the same targets. For example, Thrombin-03 exhibited a $K_{d}$ of $7.04 \mathrm{pM}$ (Fig. 3a), approximately two and three orders of magnitude superior to existing thrombin aptamers described by Bock ${ }^{[20]}(2.6 \mathrm{nM})$ and Tasset ${ }^{[21]}(5.4 \mathrm{nM})$, respectively, as measured with the same binding assay (Fig. S4). Similarly, our best ApoE aptamer (ApoE-06) exhibited a $K_{d}$ of 938 pM (Fig. 3b), four-fold better than an aptamer previously isolated by our group using high-stringency microfluidic selection ${ }^{[22]}$. Importantly, particle display also yielded high-affinity DNA aptamers for PAI-1 and 4-1BB, for which previous attempts to generate natural DNA aptamers via SELEX were unsuccessful. Our PAI-1-01 aptamer exhibits a $K_{d}$ of 339 pM (Fig. 3c) and 4-1BB-07 shows a $K_{d}$ of $2.32 \mathrm{nM}$ (Fig. 3d), both comparable to aptamers previously generated using modified bases ${ }^{[8]}$. We also obtained similar $K_{d}$ measurements using aptamers in solution to bind bead-conjugated target proteins, further validating these results (Fig. S5). 
In this work, we report a novel aptamer discovery technology that yields superior aptamers in fewer rounds in comparison to conventional selection. By transforming solution-phase aptamers into aptamer particles via emulsion PCR, we could subsequently use FACS to quantitatively measure the affinity of every displayed aptamer and individually sort them in a high-throughput manner. Our theoretical analysis revealed how the stringency of our screen can be tuned, and we identified two key experimental parameters that enable efficient isolation of the highest affinity aptamers in a given pool. Using these parameters, we generated natural DNA aptamers with excellent affinities for four different proteins in three rounds. Our aptamers for thrombin and ApoE exhibited affinities that were orders of magnitude better than previously reported values. Equally importantly, our method also yielded high-affinity natural DNA aptamers for PAI-1 and 4-1BB, two targets for which previous attempts to generate such aptamers were unsuccessful. The affinities of our aptamers were comparable to those obtained with these chemically-modified aptamers, which suggests that natural DNA aptamers may be able to target a broader swath of the proteome than previously hypothesized, with the aptamer selection strategy representing a critical limiting factor in determining success or failure for a given target.

We have identified a number of opportunities for future expansion of our technique. For example, we believe that the multicolor sorting capabilities of FACS could be exploited by labeling closely-related protein variants with distinct fluorophores as a means to generate highly selective aptamers that can discriminate subtle variations, such as posttranslational modifications or splice variants, as has been previously demonstrated with yeast display techniques ${ }^{[26]}$. Additionally, a growing number of non-natural nucleotides with diverse structures and functional groups ${ }^{[8,9,27]}$ are now available, and we anticipate that our particle display technique could be adapted to accommodate these novel nucleotides to both improve aptamer performance and further expand the accessible target space. Finally, our technique offers an opportunity to perform aptamer discovery directly in complex mixtures such as serum, cell lysate and various patient samples. The resulting aptamers could prove valuable for a host of applications in molecular diagnostics and targeted therapeutics ${ }^{[5,6,28]}$ and this is a subject of current investigation.

Given that our particle display strategy employs a pair of technologies that are readily commercially available (i.e., ePCR and FACS), we believe our technique should be broadly accessible to many research laboratories. Particle display should therefore offer a promising mechanism for generating high-quality aptamer reagents in a rapid and economical manner for the accelerated exploration and characterization of the human proteome.

\section{Supplementary Material}

Refer to Web version on PubMed Central for supplementary material.

\section{References}

1. Taussig MJ, Stoevesandt O, Borrebaeck CAK, Bradbury AR, Cahill D, Cambillau C, de Daruvar A, Dübel S, Eichler J, Frank R, et al. Nat Methods. 2007; 4:13-7. [PubMed: 17195019]

2. Marx V. Nat Methods. 2013; 10:829-833.

Angew Chem Int Ed Engl. Author manuscript; available in PMC 2015 May 05. 
3. Gloriam DE, Orchard S, Bertinetti D, Björling E, Bongcam-Rudloff E, Borrebaeck CAK, Bourbeillon J, Bradbury ARM, de Daruvar A, Dübel S, et al. Mol Cell Proteomics. 2010; 9:1-10. [PubMed: 19674966]

4. Marx V. Nat Methods. 2013; 10:703-707.

5. Cho EJ, Lee JW, Ellington AD. Annu Rev Anal Chem. 2009; 2:241-64.

6. Keefe AD, Pai S, Ellington A. Nat Rev Drug Discov. 2010; 9:537-50. [PubMed: 20592747]

7. Baird GS. Am J Clin Pathol. 2010; 134:529-31. [PubMed: 20855632]

8. Gold L, Ayers D, Bertino J, Bock C, Bock A, Brody EN, Carter J, Dalby AB, Eaton BE, Fitzwater T, et al. PLoS One. 2010; 5:e15004. [PubMed: 21165148]

9. Kimoto M, Yamashige R, Matsunaga K-I, Yokoyama S, Hirao I. Nat Biotechnol. 2013; 31:453-7. [PubMed: 23563318]

10. Aquino-Jarquin G, Toscano-Garibay JD. Int J Mol Sci. 2011; 12:9155-71. [PubMed: 22272125]

11. Irvine D, Tuerk C, Gold L. J Mol Biol. 1991; 222:739-61. [PubMed: 1721092]

12. Wang J, Rudzinski JF, Gong Q, Soh HT, Atzberger PJ. PLoS One. 2012; 7:e43940. [PubMed: 22952815]

13. Djordjevic M. Biomol Eng. 2007; 24:179-89. [PubMed: 17428731]

14. Kang J, Lee MS, Gorenstein DG. J Biochem Biophys Methods. 2005; 64:147-51. [PubMed: 16009429]

15. Boder ET, Wittrup KD. Nat Biotechnol. 1997; 15:553-7. [PubMed: 9181578]

16. Francisco JA. Proc Natl Acad Sci. 1993; 90:10444-10448. [PubMed: 8248129]

17. Mayer G, Ahmed MSL, Dolf A, Endl E, Knolle PA, Famulok M. Nat Protoc. 2010; 5:1993-2004. [PubMed: 21127492]

18. Dressman D, Yan H, Traverso G, Kinzler KW, Vogelstein B. Proc Natl Acad Sci U S A. 2003; 100:8817-22. [PubMed: 12857956]

19. Herzenberg LA, Tung J, Moore WA, Herzenberg LA, Parks DR. Nat Immunol. 2006; 7:681-5. [PubMed: 16785881]

20. Bock LC, Griffin LC, Latham JA, Vermaas EH, Toole JJ. Nature. 1992; 355:564-6. [PubMed: 1741036]

21. Tasset DM, Kubik MF, Steiner W. J Mol Biol. 1997; 272:688-98. [PubMed: 9368651]

22. Ahmad KM, Oh SS, Kim S, McClellen FM, Xiao Y, Soh HT. PLoS One. 2011; 6:e27051. [PubMed: 22110600]

23. Blake CM, Sullenger BA, Lawrence DA, Fortenberry YM. Oligonucleotides. 2009; 19:117-28. [PubMed: 19284310]

24. McNamara JO, Kolonias D, Pastor F, Mittler RS, Chen L, Giangrande PH, Sullenger B, Gilboa E. J Clin Invest. 2008; 118:376-86. [PubMed: 18060045]

25. Zuker M. Nucleic Acids Res. 2003; 31:3406-15. [PubMed: 12824337]

26. Garcia-Rodriguez C, Levy R, Arndt JW, Forsyth CM, Razai A, Lou J, Geren I, Stevens RC, Marks JD. Nat Biotechnol. 2007; 25:107-16. [PubMed: 17173035]

27. Keefe AD, Cload ST. Curr Opin Chem Biol. 2008; 12:448-56. [PubMed: 18644461]

28. Gold L, Janjic N, Jarvis T, Schneider D, Walker JJ, Wilcox SK, Zichi D. Cold Spring Harb Perspect Biol. 2012; 4 

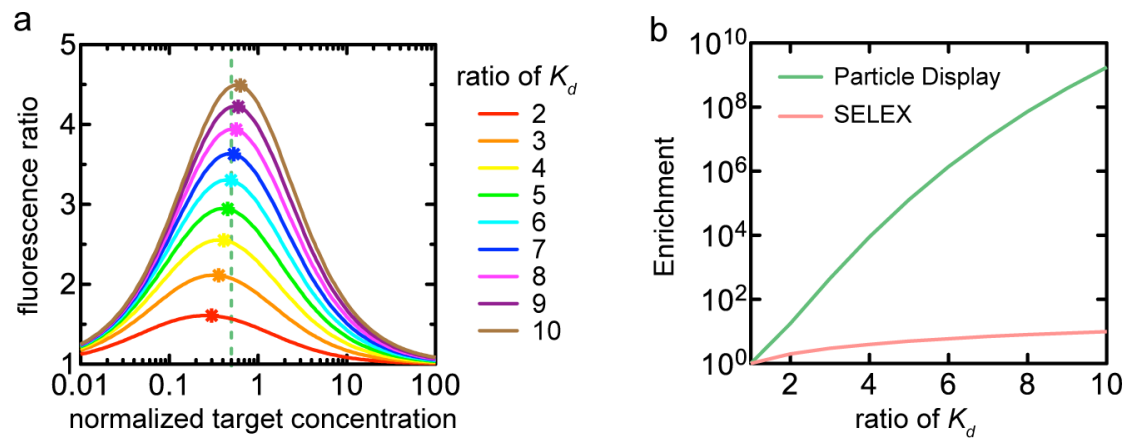

Figure 1.

Theoretical analysis of the particle display system. (a) Fluorescence ratios between APs displaying the highest-affinity aptamer and a lower-affinity aptamer are plotted as a function of normalized target concentration (relative to the $K_{d}$ of the highest-affinity aptamer) for multiple $K_{d}$ ratios ranging from 2 to 10 . A target concentration equal to $50 \%$ of the $K_{d}$ of the highest-affinity aptamer (vertical dashed line) ensures that the fluorescence difference is within $2 \%$ of the optimal value (denoted by $*$ ) across this entire range of $K_{d}$ ratios, (b) Calculated enrichment performance of particle display exceeds the theoretical maximum of SELEX by many orders of magnitude. 


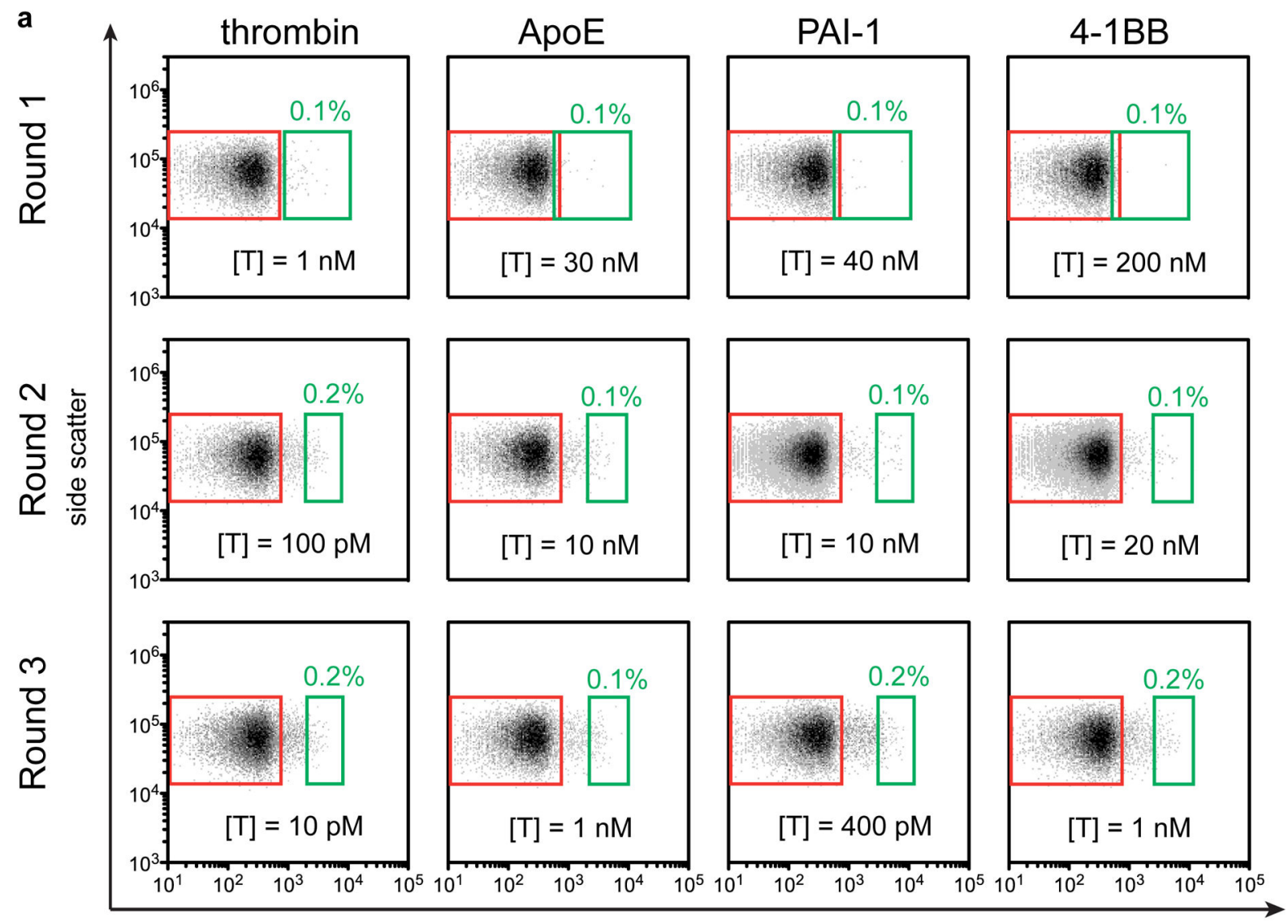

fluorescence intensity

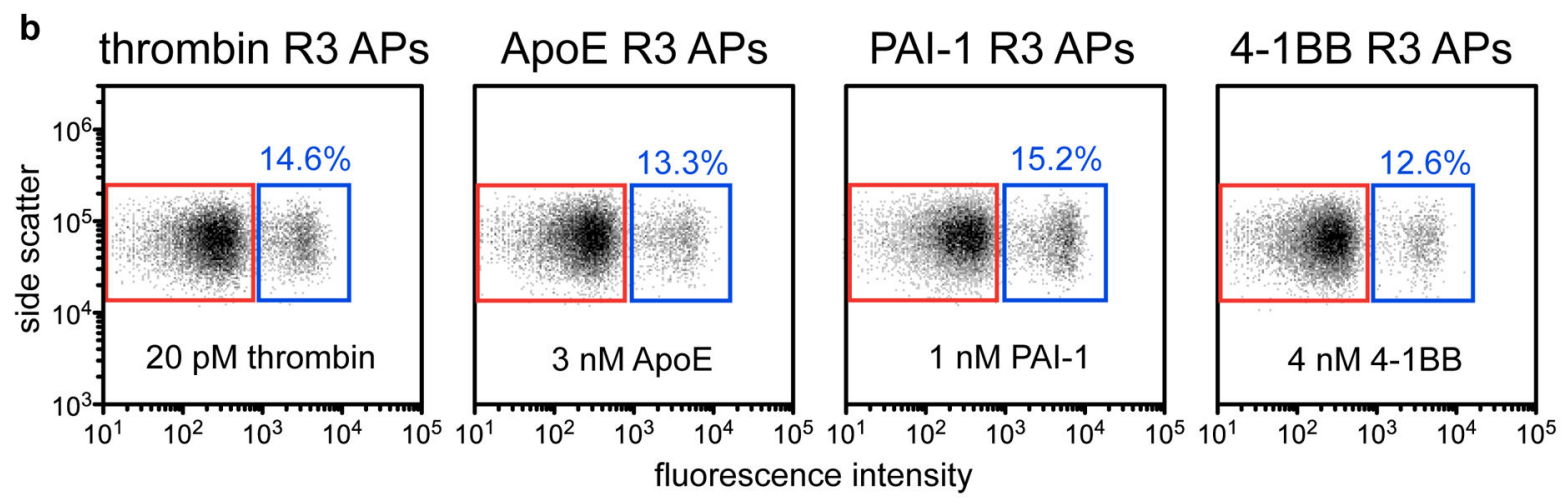

Figure 2.

Particle display screening for four protein targets. (a) FACS plots with sort gates for three screening rounds at different target concentrations ([T]). APs (represented by individual dots) residing within reference gates (red) displayed either FP (negative controls) or nonbinding sequences, while APs residing within the sort gate (green) displayed aptamers with desired affinity and were collected and used as templates for the subsequent round. (b) Aptamers isolated in R3 exhibited high affinities for their target protein. Relative to the starting pool, a far greater percentage of the R3 AP population showed high levels of target binding even at considerably lower target concentrations, indicating greatly increased affinity. 

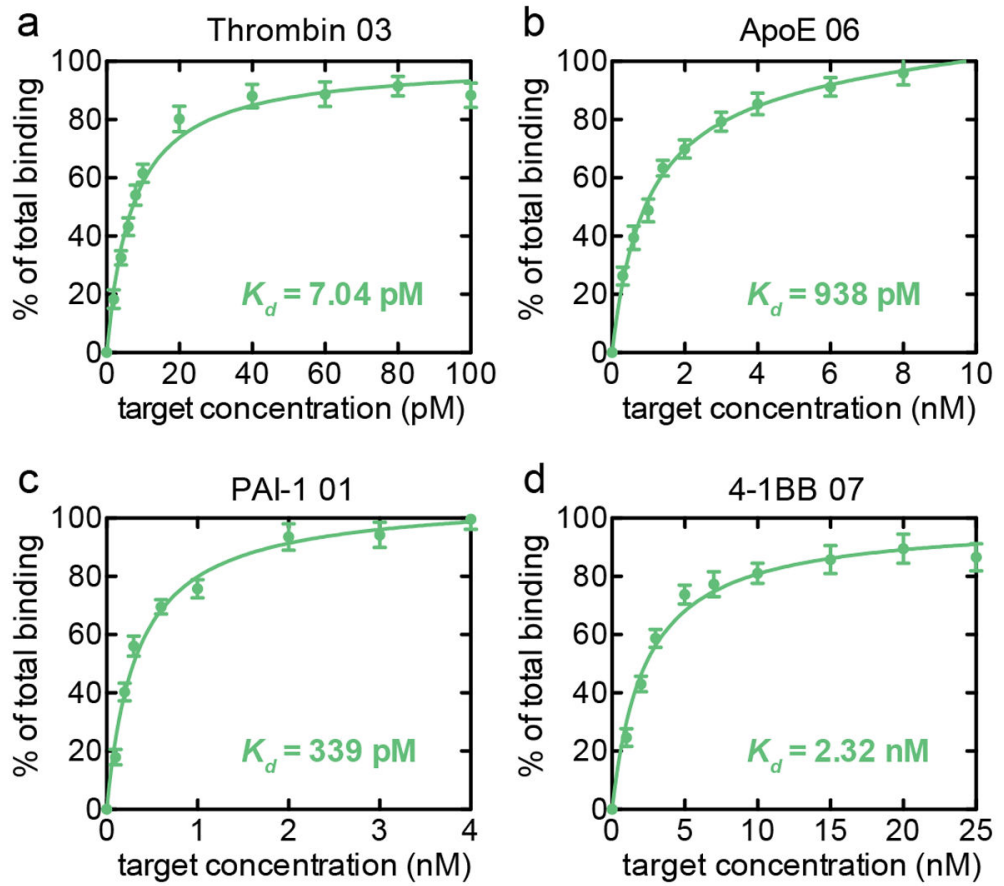

Figure 3.

Highest affinity aptamers obtained via particle display for four protein targets: (a) thrombin, (b) ApoE, (c) PAI-1 and (d) 4-1BB. We calculated $\mathrm{K}_{\mathrm{d}}$ using a Langmuir 1:1 binding model. 


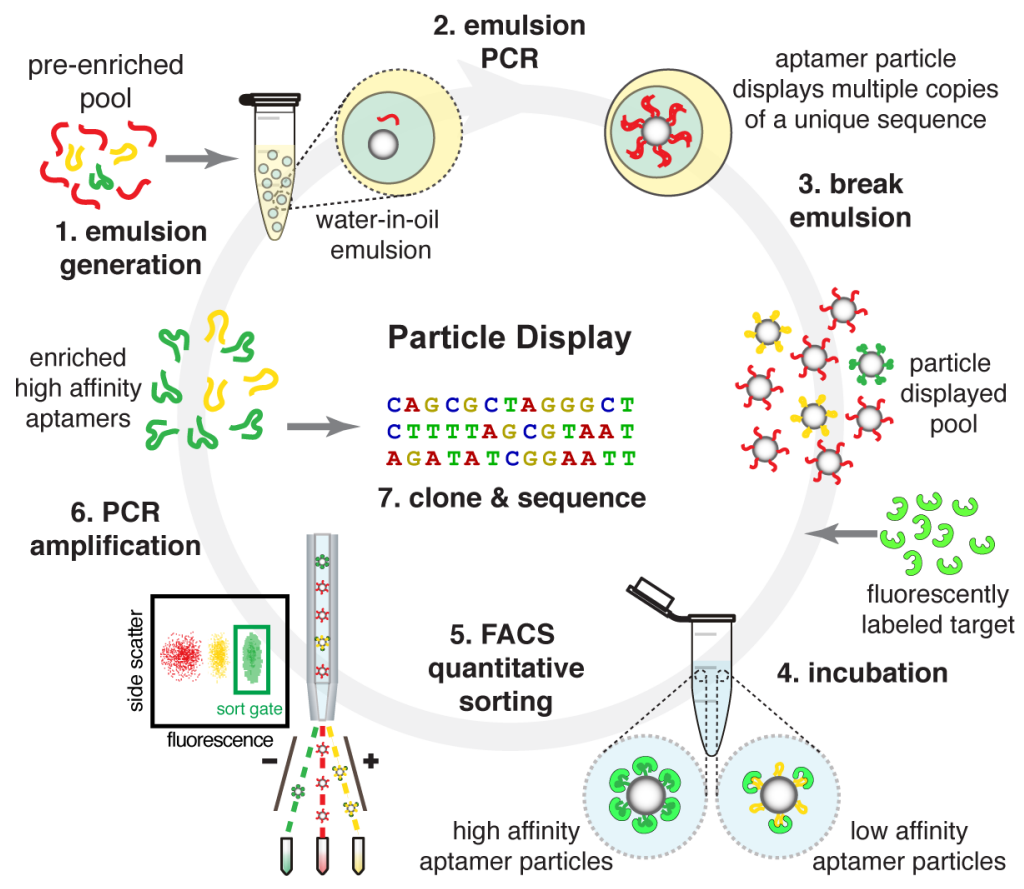

Scheme 1.

Particle display system overview. After an initial round of pre-enrichment via conventional screening to reduce the diversity of starting library, APs are synthesized by emulsion PCR, yielding particles that each display $10^{5}$ copies of a single aptamer sequence (step 1-3).

These are then incubated with fluorescently-labeled target molecules (step 4) and quantitatively screened by FACS (step 5) to isolate high-affinity aptamers based on increased fluorescence intensity. These AP-displayed aptamers are then PCR amplified to generate an enriched pool (step 6), which is either sequenced (step 7) or used to synthesize APs for the next round of screening (step 1). 